\title{
A case-control study of vitamin D status and asthma in adults
}

\author{
K. Allan ${ }^{1}$, G. Devereux ${ }^{1}$, G. McNeill ${ }^{1}$, A. Wilson ${ }^{2}$, A. Avenell ${ }^{1}$ and W. Fraser ${ }^{3}$ \\ ${ }^{1}$ Institute of Applied Health Sciences, University of Aberdeen, Aberdeen AB25 2ZD, UK, ${ }^{2}$ Biomedicine Group, School of \\ Medicine, University of East Anglia, Norwich, NR4 7TJ, UK and ${ }^{3}$ Metabolic Bone Diseases Unit, Department of Clinical \\ Chemistry, University of Liverpool, Duncan Building, Daulby St, Liverpool L69 3GA, UK
}

It has been suggested that the rapid increase in the prevalence of asthma in developed countries in recent decades may be the result of changes in diet and lifestyle ${ }^{(1)}$. Asthma is associated with an alteration in the balance of T-helper lymphocytes with an increase in proinflammatory Th2 cells. As vitamin D may directly suppress Th2 differentiation ${ }^{(2,3)}$, it has been hypothesised that low vitamin D status, as a consequence of sun avoidance behaviours and an increasingly indoor lifestyle, could contribute to the rising prevalence of asthma ${ }^{(4)}$.

The present study was designed to compare the vitamin D status of age and sex-matched adults with and without physician-confirmed asthma. The study was conducted in the Chest Clinic, Aberdeen Royal Infirmary and the Department of Respiratory Medicine; Norfolk and Norwich University Hospital, Norfolk. One hundred and sixty participants aged between 18 and 50 years were recruited, 80 with physician-confirmed mild/moderate asthma and 80 age and gender-matched controls. Cases and controls were assessed within a month of each other to control for seasonal variation of sunlight exposure. Controls were individuals without asthma who had a smoking history of $<10$ pack-years. The majority of controls $(70 \%)$ were recruited from local daycase surgery units, the remainder bring recruited after advertising in local press. Ninety-four participants were recruited in Aberdeen between June 2007 and April 2008, and 66 in Norwich between October 2007 and September 2008. Vitamin D status was assessed by serum 25-hydroxyvitamin $\mathrm{D}_{3}$ measured by HPLC-tandem mass spectrometry.

Mean serum 25-hydroxyvitamin $\mathrm{D}_{3}$ concentration was $8.68 \mathrm{ng} / \mathrm{ml}(95 \%$ CI 7.60, 9.75), being lower in Aberdeen $6.78 \mathrm{ng} / \mathrm{ml}(95 \% \mathrm{CI}$ $5.32,8.25)$ than Norwich $11.5 \mathrm{ng} / \mathrm{ml}(95 \%$ CI 10.2, 12.8). In Aberdeen, $76 \%$ of the participants had serum levels below the generally accepted cut-off for a deficiency of $10 \mathrm{ng} / \mathrm{ml}^{(5)}$. In Norwich, this figure was $42 \%$. In winter (December-February), these proportions rose to $92.3 \%$ and $46.4 \%$, respectively. There was no significant difference in the serum 25 -hydroxyvitamin $\mathrm{D}_{3}$ concentrations between cases and controls: $8.50 \mathrm{ng} / \mathrm{ml}$ (95\% CI 7.06, 9.95) v. 8.86 (95\% CI 7.22, 10.5). Conditional logistic regression adjusting serum 25-hydroxyvitamin $\mathrm{D}_{3}$ levels for age, gender, smoking status, BMI and season of assessment revealed no difference in serum 25-hydroxyvitamin $\mathrm{D}_{3}$ levels between cases and controls (OR asthma $v$. control 0.98 (95\% CI 0.91, 1.04), $P=0.50$ ). Similar multivariable analysis demonstrated association neither between 25-hydroxyvitamin $\mathrm{D}_{3}$ levels and asthma severity nor lung function (FEV $\%$ predicted).

This study does not find evidence to support the use of vitamin D as an adjunct to conventional therapy in asthma in adults.

This study was funded by NHS Grampian Endowment Funds.

1. Devereux G (2006) The increase in the prevalence of asthma and allergy: food for thought. Nat Rev Immunol 6, 869-874.

2. Jirapongsananuruk O, Melamed I \& Leung DY (2000) Additive immunosuppressive effects of 1,25-dihydroxyvitamin $\mathrm{D}_{3}$ and corticosteroids on TH1, but not TH2, responses. J Allergy Clin Immunol 106, 981-985.

3. Urry Z, Xystrakis E, Richards DF et al. (2009) Ligation of TLR9 induced on human IL-10-secreting Tregs by 1alpha,25-dihydroxyvitamin $\mathrm{D}_{3}$ abrogates regulatory function. J Clin Invest 119, 387-398.

4. Litonjua AA \& Weiss ST (2007) Is vitamin D deficiency to blame for the asthma epidemic? J Allergy Clin Immunol 120, 1031-1035.

5. Devereux G, MacDonald H \& Hawrylowicz C (2009) Vitamin D and asthma time for intervention? Am J Respir Crit Care Med 179, 739-742. 